\title{
A Deterministic Single-Photon Source for Distributed Quantum Networking
}

\author{
Axel Kuhn, Markus Hennrich, and Gerhard Rempe \\ Max-Planck-Institut für Quantenoptik, Hans-Kopfermann-Str.1, 85748 Garching, Germany
}

(Dated: February 9, 2008)

\begin{abstract}
A sequence of single photons is emitted on demand from a single three-level atom strongly coupled to a high-finesse optical cavity. The photons are generated by an adiabatically driven stimulated Raman transition between two atomic ground states, with the vacuum field of the cavity stimulating one branch of the transition, and laser pulses deterministically driving the other branch. This process is unitary and therefore intrinsically reversible, which is essential for quantum communication and networking, and the photons should be appropriate for all-optical quantum information processing.
\end{abstract}

PACS numbers: 03.67.-a, 03.67.Hk, 42.55.Ye, 42.65.Dr

A future quantum network connecting remote quantum processors and memories has several advantages in processing quantum information as compared to a local quantum computer, since it combines scalability with modularity. Different kinds of networks have been proposed [1]: one is an all-optical network [2], where the nodes are linear optical components, with quantum information encoded in the number of photons flying from node to node. The nodes perform gate operations based on quantum interference effects between indistinguishable photons. In another, more general, network the nodes also serve as quantum memories storing information, e.g., in long-lived states of atoms located in an optical cavity [3]. The key requirement for such a network is its ability to interconvert stationary and flying qubits and to transmit flying qubits between specified locations [ 4 . The atom-cavity system, in particular, must be able to transfer quantum information between atoms and photons in a coherent manner [5, 6. It must also act as an emitter and a receiver of single-photon states. These states must therefore be generated by a reversible process. However, all deterministic single-photon emitters demonstrated so far [7, 8, 9, 10, 11, 12, 13, 14, 15] do not meet this essential requirement. The reason is that the emission process, namely an electronic excitation of the system followed by spontaneous emission, cannot be described by a Hamiltonian evolution and, hence, is irreversible.

This letter describes the realization of an intrinsically reversible single-photon source [3, 16, 17, 18, 19], which is based on a stimulated Raman process driving an adiabatic passage [20] (STIRAP) between two ground states of a single atom strongly coupled to a single mode of a high-finesse optical cavity [21, 22]. A laser beam illuminating the atom excites one branch of the Raman transition, while the cavity vacuum stimulates the emission of the photon on the other branch. STIRAP is slow compared to the photon lifetime in the cavity, so that the field generated inside the cavity is instantaneously mapped to the outside world. Moreover, it employs a dark state, which has two important consequences: first, any electronic excitation is avoided, so that irreversible

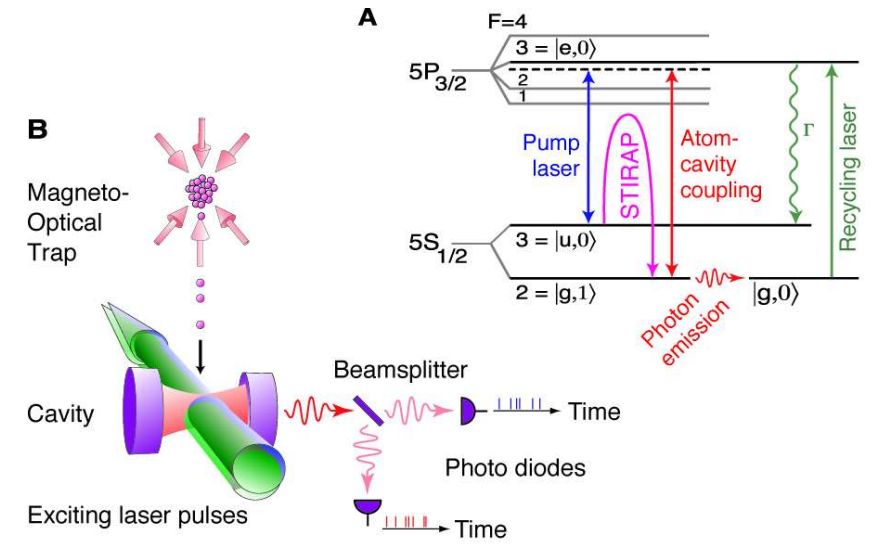

FIG. 1: Scheme of the experiment. (A) Relevant energy levels and transitions in ${ }^{85} \mathrm{Rb}$. The atomic states labeled $|u\rangle,|e\rangle$ and $|g\rangle$ are involved in the Raman process, and the states $|0\rangle$ and $|1\rangle$ denote the photon number in the cavity. (B) Setup: A cloud of atoms is released from a magneto-optical trap and falls through a cavity $20 \mathrm{~cm}$ below in about $8 \mathrm{~ms}$ with a velocity of $2 \mathrm{~m} / \mathrm{s}$. The interaction time of a single atom with the $\mathrm{TEM}_{00}$ mode of the cavity (waist $w_{0}=35 \mu \mathrm{m}$ ) amounts to about $17.5 \mu \mathrm{s}$. The pump and recycling lasers are collinear and overlap with the cavity mode. Photons emitted from the cavity are detected by a pair of photodiodes with a quantum efficiency of $50 \%$.

spontaneous processes do not occur. Second, the scheme allows one to continuously tune the frequency of the photon within a range that is only limited by the atom-cavity coupling strength. The tuning ability has recently been demonstrated with a beam of atoms passing through the cavity [23]. This experiment produced at most one photon per passing atom, but did not operate as a singlephoton source, because its continuous driving scheme simply mapped the random (Poissonian) atom statistics to the photons. The present experiment, however, uses a pulsed driving together with a pulsed recycling. This makes possible to produce on demand a stream of several single-photon pulses from one-and-the-same atom, triggered by the detection of a "first" photon emitted from the cavity.

Figure 11A shows the basic scheme of the photon- 
generation process. A single ${ }^{85} \mathrm{Rb}$ atom is prepared in state $|u\rangle$, which is the $F=3$ hyperfine state of the $5 S_{1 / 2}$ electronic ground state. The atom is located in a highfinesse optical cavity, which is near resonant with the $780 \mathrm{~nm}$ transition between states $|g\rangle$ and $|e\rangle$. Here, $|g\rangle$ is the $F=2$ hyperfine state of the electronic ground state and $|e\rangle$ is the electronically excited $5 P_{3 / 2}(F=3)$ state. The state of the cavity is denoted by $|n\rangle$, where $n$ is the number of photons. When the atom is placed inside the cavity, the product states $|g, n\rangle$ and $|e, n-1\rangle$ are coupled by the electric dipole interaction, characterized by the Rabi frequency $\Omega_{n}=2 g \sqrt{n}$. Here, $g$ is the average atom-cavity coupling constant, which takes into account that neither the position of the atom in the cavity, nor the magnetic quantum number of the atom is well defined in the experiment. We assume $g$ to be constant while a pump-laser pulse with Rabi frequency $\Omega_{P}(t)$ is applied. This laser is close to resonance with the $|u\rangle \leftrightarrow|e\rangle$ transition, so that now the three product states $|u, n-1\rangle,|e, n-1\rangle$ and $|g, n\rangle$ of the atom-cavity system are coupled. For the one-photon manifold, $n=1$, and a Raman-resonant excitation, where the detunings of the pump laser, $\Delta_{P}$, and the cavity, $\Delta_{C}$, from the respective atomic transitions are equal, it is straightforward to find the three eigenstates of the coupled atom-cavity system, $\left|\phi_{1}^{ \pm}\right\rangle$and $\left|\phi_{1}^{0}\right\rangle=\left[2 g|u, 0\rangle-\Omega_{P}(t)|g, 1\rangle\right] / \sqrt{4 g^{2}+\Omega_{P}^{2}(t)}$. Note that state $\left|\phi_{1}^{0}\right\rangle$ is dark, i.e. has no contribution of the excited state, $|e\rangle$, and is therefore not affected by spontaneous emission.

The dark state $\left|\phi_{1}^{0}\right\rangle$ is now used to generate a single photon inside the cavity. This is achieved by establishing a large atom-cavity coupling constant, $g$, before turning on the pump pulse. In this case, the system's initial state, $|u, 0\rangle$, coincides with $\left|\phi_{1}^{0}\right\rangle$. Provided the pump pulse rises slowly, the system's state vector adiabatically follows any change of $\left|\phi_{1}^{0}\right\rangle$, and for a lossless cavity a smooth transition from $|u, 0\rangle$ to $|g, 1\rangle$ is realized as soon as $\Omega_{P} \gg 2 g$. Hence, a single photon is generated in the relevant cavity mode. This photon leaves the cavity through that mirror which is designed as an output coupler. The emission starts as soon as the decaying state, $|g, 1\rangle$, contributes to $\left|\phi_{1}^{0}\right\rangle$, i.e. already with the rising edge of the pump pulse, because the contribution from $|g, 1\rangle$ is proportional to $\Omega_{P}^{2}(t)$. If the pump pulse rises slowly, the emission can therefore end even before $\Omega_{P}>2 g$. The dynamics of the simultaneous excitation and emission processes determines the duration and, hence, the linewidth of the photon. When the photon is emitted, the final state of the coupled system, $|g, 0\rangle$, is reached. This state is not coupled to the one-photon manifold, and the atom cannot be reexcited. This limits the number of photons per pump pulse and atom to one.

To emit a sequence of photons from one-and-the-same atom, the system must be transferred back to $|u, 0\rangle$ once an emission has taken place. To do so, we apply recycling laser pulses that hit the atom between consecutive

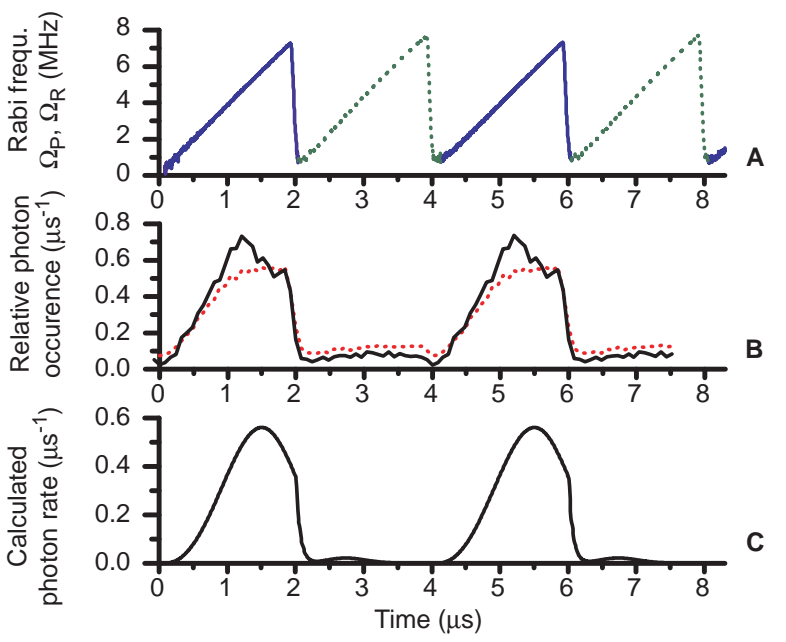

FIG. 2: Pulse shapes. (A) The atoms are periodically illuminated with $2 \mu$ s-long pulses from the pump (solid line) and the recycling laser (dotted line). (B) Measured arrivaltime distribution of photons emitted from the cavity (dotted line). The solid line shows the arrival-time distribution of photons emitted from strongly coupled atoms (see text). (C) Simulation of the process with $\left(g, \Omega_{P, R}^{0}, \Delta_{P, C}, \Gamma, \kappa\right)=$ $2 \pi \times(2.5,8.0,-20.0,6.0,1.25) \mathrm{MHz}$, where $\Omega_{P, R}^{0}$ are the peak Rabi frequencies of the pump- and recycling pulses, and $\Gamma$ and $\kappa$ are the atom and cavity-field decay rates, respectively.

pump pulses. The recycling pulses are resonant with the $|g\rangle \leftrightarrow|e\rangle$ transition and pump the atom to state $|e\rangle$. From there, it decays spontaneously to the initial state, $|u\rangle$. Note that state $|e\rangle$ populated by the recycling laser couples to the cavity. However, spontaneous emission into the cavity is suppressed by deliberately choosing a large cavity detuning, $\Delta_{C}$. The pump laser is detuned by the same amount to assure Raman resonance. If an atom that resides in the cavity is now exposed to a sequence of laser pulses, which alternate between triggering singlephoton emissions and re-establishing the initial condition by optical pumping, a sequence of single-photon pulses is produced.

Figure 11B shows the apparatus. Atoms are released from a magneto-optical trap and pass through the $\mathrm{TEM}_{00}$ mode of the optical cavity, where they are exposed to the sequence of laser pulses. On average, 3.4 atoms/ms enter the cavity [28], so that the probability of finding a single atom inside the cavity is $5.7 \%$, while the probability of having more than one atom is only $0.18 \%$ which is negligible. The cavity is $1 \mathrm{~mm}$ long and has a finesse of 60000 . One mirror has a 25 times larger transmission coefficient than the other. Therefore, photons are preferentially emitted into one direction. These photons are counted by two avalanche photodiodes which are placed at the output ports of a beam splitter. For each experimental cycle, all photon arrival times are recorded with transient digitizers with a time resolution of $8 \mathrm{~ns}$.

In the experiment, the electric field amplitudes and, 


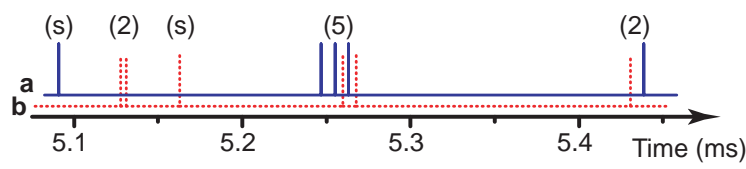

FIG. 3: Photon sequence: Clip of the photon streams arriving at the photodiodes D1 and D2 (traces $\mathbf{a}$ and $\mathbf{b}$, respectively). Several sequences of two (2) and five (5) photon emissions are observed, with durations comparable to the atom-cavity interaction time. The solitary events (s) are either dark counts, or, more likely, photons coming from atoms that are only weakly coupled to the cavity.

hence, the Rabi frequencies of the pump and recycling pulses have the shape of a saw-tooth and increase linearly, as displayed in Fig. 2A. This leads to a constant rate of change of the dark state, $\left|\phi_{1}^{0}\right\rangle$, during the initial stage of the pump pulses, and therefore optimal adiabaticity with minimal losses to the other eigenstates. The linear slope of the recycling pulses suppresses higher Fourier components and therefore reduces photon emission into the detuned cavity. Note that the recycling process is finished before the end of the pulse is reached, so that the final sudden drop in Rabi frequency does not influence the atom.

Also shown in Fig.2 2 are two measured arrival-time distributions of the photons and a simulation of the photon emission rate for typical experimental parameters. The simulation is based on a numerical solution of the system's master equation 22] which takes into account the decay of the relevant states. The simulation (Fig. 22C) reveals that the pump-pulse duration of $2 \mu$ s is slightly too short, as the emitted photon pulse is not completely finished. This is also observed in the photon arrival-time distribution (Fig.2B). Here, the measured data agree well with the simulation if only photons from strongly coupled atoms are considered (solid line). For these, we assume that several photons are detected within he atom-cavity interaction time. If solitary photons, which we attribute to weakly coupled atoms, are included in the analysis, the arrival-time distribution is given by the dotted line. Note that the envelope of the photon pulses is well explained by the expected shape of the single-photon wavepackets, and therefore cannot be attributed to an uncertainty in emission time, which is not present for a unitary process. Assuming transform-limited Gaussian pulses, we infer a single-photon linewidth of $\Delta \nu=340 \mathrm{kHz}$ (FWHM) from the $1.3 \mu \mathrm{s}$ photon-pulse duration (FWHM). We emphasize that the pump-pulse duration was adjusted to maximize the number of photons per atom. Longer pump pulses would not truncate the photon pulses and, hence, would slightly increase the emission probability per pulse, but due to the limited atom-cavity interaction time, the total number of photons per atom would be reduced.

Figure 3 displays an example of the photon stream recorded while single atoms fall through the cavity one

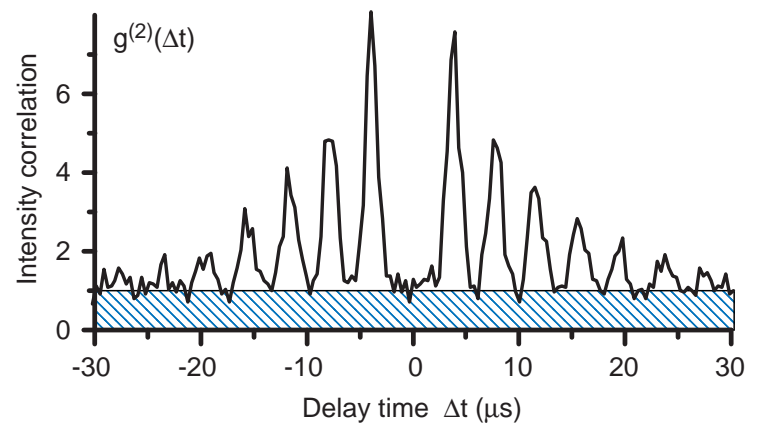

FIG. 4: Second-order intensity correlation of the emitted photon stream, averaged over 15000 experimental cycles (loading and releasing of the atom cloud) with a total number of 184868 photon counts. The hatched area represents correlations between photons and detector-noise counts.

after the other. Obviously, the photon sequence is different for each atom. In particular, not every pump pulse leads to a detected photon, since the efficiencies of photon generation and photon detection are limited. The second-order intensity correlation function of the emitted photon stream is shown in Fig. 1. Displayed is the cross-correlation of the photon streams registered by the two photodiodes $D 1$ and $D 2$. It is defined as $g^{(2)}(\Delta t)=\left\langle P_{D 1}(t) P_{D 2}(t-\Delta t)\right\rangle /\left(\left\langle P_{D 1}(t)\right\rangle\left\langle P_{D 2}(t)\right\rangle\right)$, where $P_{D 1}(t)$ and $P_{D 2}(t)$ are the probabilities to detect a photon at time $t$ with photodiode $D 1$ and $D 2$, respectively. Note that all photon-arrival times are recorded to calculate the full correlation function, without the otherwise usual restriction of a simple start/stop measurement which would consider only neighboring events. Of course, $g^{(2)}$ includes not only correlations between photons emitted from the cavity but also those involving detector-noise counts. This last contribution has been determined from an independent measurement of the detector-noise count rate. The result is indicated by the time-independent hatched area in Fig. 因. Only the excess signal, $\tilde{g}^{(2)}(\Delta t)=g^{(2)}(\Delta t)-g_{\text {noise }}^{(2)}$, reflects the true photon statistics of the light emitted from the atom-cavity system.

The correlation function, $\tilde{g}^{(2)}(\Delta t)$, oscillates with the same periodicity as the sequence of pump pulses. This indicates that photons are only emitted during the pump pulses, and no emissions occur when recycling pulses are applied. The nearly Gaussian envelope of the comb-like function is obviously a consequence of the limited atomcavity interaction time. The most remarkable feature in Fig. 1 is the missing correlation peak at $\Delta t=0$. In fact, photon antibunching together with $\tilde{g}^{(2)}(0) \approx 0$ is observed. This clearly demonstrates the nonclassical character of the emitted light, and proofs that (a) the number of emitted photons per pump pulse is limited to one, and (b) no further emission occurs before the atom is recycled to its initial state. Note that the relatively large 
noise contribution is no intrinsic limitation of our system but reflects only the low atomic flux through the cavity in the present experiment.

We emphasize that the detection of a first photon signals the presence of an atom in the cavity, and fixes the atom number to one. The photons emitted from this atom during subsequent pump pulses dominate the photon statistics and give rise to antibunching. Such an antibunching would not be observed for faint laser pulses, since a random photon statistics applies to each pulse. The areas of the different peaks of the correlation function in Fig. 4 reflect the probability for the emission of further photons from one-and-the-same atom. They are determined from a lengthy but straightforward calculation, which relates the number of correlations per pulse with the total number of photons. Using the data displayed in Fig. 4, the result for the conditional emission of another photon during the (next, $3^{r d}, 4^{t h}, 5^{t h}, 6^{t h}$, $7^{\text {th }}$ ) pump pulse is $(8.8,5.1,2.8,1.4,0.8,0.5) \%$. Note that the probabilities for subsequent emissions decrease, since the photon emission probability, $P_{\text {emit }}$, depends on the location of the moving atom. It is highest for an atom in an antinode, and decreases if the atom moves away from this point. It is not possible to control the atoms location in the present experiment, but it is possible to calculate $P_{\text {emit }}(z)$ from the experimental data. Here, $z$ is the atom's vertical position relative to the cavity axis, and $P_{\text {emit }}(z)$ is averaged over all possible atomic trajectories in the horizontal $x y$-plane. Assuming a Gaussian $z$-dependence, the deconvolution of $\tilde{g}^{(2)}(\Delta t)$ gives $P_{\text {emit }}(z)=0.17 \exp \left[-(z / 15.7 \mu m)^{2}\right]$. For $z=0$, the average photon-emission probability of $17 \%$ is smaller than the calculated value of $67 \%$ for an atom in an antinode of the cavity. It follows that a system combining a cavity and a single atom at rest in a dipole trap [24, 25, or a single ion at rest in a rf-trap [26, 27, should allow one to generate a continuous bit-stream of single photons with a large and time-independent efficiency [21, 22]. The photon repetition rate is limited by the atom-cavity coupling constant, $g$, which one could push into the $\mathrm{GHz}$ regime by using smaller cavities of wavelength-limited dimensions in, e.g., a photonic bandgap material.

In conclusion, we have shown that a coupled atomcavity system is able to emit single photons on demand. Moreover, it is possible to generate a sequence of up to seven photons on demand from one-and-the-same atom in a time interval of about $30 \mu \mathrm{s}$. These photons are all generated in a well-defined radiation mode. They should have the same frequency and a Fourier-transform limited linewidth, limited from above by the decay rate of the cavity field 23]. It follows that one can expect the photons to be indistinguishable and, therefore, ideal for alloptical quantum computation schemes [2]. Moreover, the photon-generation process is unitary. This makes possible to produce arbitrarily shaped single-photon pulses by suitably tailoring the envelope of the pump pulse. For symmetric pulses, the emission process can be reversed. This should allow one to transfer the photon's quantum state to another atom located in another cavity. Such a state mapping between atoms and photons is the key to quantum teleportation of atoms between distant nodes in a quantum network of optical cavities [3].

This work was supported by the research program 'Quantum Information Processing' of the Deutsche Forschungsgemeinschaft, and by the European Union through the IST (QUBITS) and IHP (QUEST) programs.

[1] see, e.g. C. Monroe, Nature 416, 238 (2002).

[2] E. Knill, R. Laflamme, and G. J. Milburn, Nature 409, 46 (2001).

[3] J. I. Cirac, P. Zoller, H. J. Kimble, and H. Mabuchi, Phys. Rev. Lett. 78, 3221 (1997).

[4] D. P. DiVincenzo, Fortschr. Phys. 48, 771 (2000).

[5] X. Maître et al. Phys. Rev. Lett. 79, 769 (1997).

[6] S. Brattke, B. T. H. Varcoe, and H. Walther, Phys. Rev. Lett. 86, 3534 (2001).

[7] J. Kim, O. Benson, H. Kan, and Y. Yamamoto, Nature 397, 500 (1999).

[8] B. Lounis and W. E. Moerner, Nature 407, 491 (2000).

[9] C. Kurtsiefer, S. Mayer, P. Zarda, and H. Weinfurter, Phys. Rev. Lett. 85, 290 (2000).

[10] R. Brouri, A. Beveratos, J.-P. Poizat, and P. Grangier, Opt. Lett. 25, 1294 (2000).

[11] P. Michler et al. Nature 406, 968 (2000).

[12] C. Santori et al. Phys. Rev. Lett. 86, 1502 (2001).

[13] Z. Yuan et al. Science 295, 102 (2002).

[14] P. Michler et al. Science 290, 2282 (2000).

[15] E. Moreau et al. Appl. Phys. Lett. 79, 2865 (2001).

[16] S. van Enk et al. J. Mod. Opt. 44, 1727 (1997).

[17] A. S. Parkins, P. Marte, P. Zoller, and H. J. Kimble, Phys. Rev. Lett. 71, 3095 (1993).

[18] A. S. Parkins et al. Phys. Rev. A 51, 1578 (1995).

[19] T. Pellizari, S. A. Gardiner, J. I. Cirac, and P. Zoller, Phys. Rev. Lett. 75, 3788 (1995).

[20] N. V. Vitanov, M. Fleischhauer, B. W. Shore, and K. Bergmann, Adv. At. Mol. Opt. Phys. 46, 55 (2001).

[21] C. K. Law and H. J. Kimble, J. Mod. Opt. 44, 2067 (1997).

[22] A. Kuhn, M. Hennrich, T. Bondo, and G. Rempe, Appl. Phys. B 69, 373 (1999).

[23] M. Hennrich, T. Legero, A. Kuhn, and G. Rempe, Phys. Rev. Lett. 85, 4872 (2000).

[24] N. Schlosser, G. Reymond, I. Protsenko, and P. Grangier, Nature 411, 1024 (2001).

[25] S. Kuhr et al. Science 293, 278 (2001).

[26] G. R. Guthörlein et al. Nature 414, 49 (2001).

[27] A. B. Mundt et al. Phys. Rev. Lett.(to be published), quant-ph/0202112.

[28] The flux of atoms is determined by a statistical analysis of the emitted light, with continuous pump- and recycling lasers exciting the falling atoms. As the cavity acts as detector, non-interacting atoms are ignored, so that its spatial mode structure is taken into account. 